University of New Hampshire

University of New Hampshire Scholars' Repository

Space Science Center

Institute for the Study of Earth, Oceans, and

Space (EOS)

3-10-2003

\title{
Hard x-ray polarimeter for gamma-ray bursts and solar flares
}

\author{
Mark L. McConnell \\ University of New Hampshire - Main Campus, mark.mcconnell@unh.edu \\ J Ledoux \\ University of New Hampshire - Main Campus \\ John R. Macri \\ University of New Hampshire - Main Campus, John.Macri@unh.edu \\ James M. Ryan \\ University of New Hampshire, James.Ryan@unh.edu
}

Follow this and additional works at: https://scholars.unh.edu/ssc

Part of the Astrophysics and Astronomy Commons

\section{Recommended Citation}

Mark L. McConnell ; James Ledoux ; John R. Macri and James M. Ryan "Hard x-ray polarimeter for gamma-ray bursts and solar flares", Proc. SPIE 4851, X-Ray and Gamma-Ray Telescopes and Instruments for Astronomy, 1382 (March 10, 2003); doi:10.1117/12.461411; http://dx.doi.org/10.1117/12.461411

This Conference Proceeding is brought to you for free and open access by the Institute for the Study of Earth, Oceans, and Space (EOS) at University of New Hampshire Scholars' Repository. It has been accepted for inclusion in Space Science Center by an authorized administrator of University of New Hampshire Scholars' Repository. For more information, please contact Scholarly.Communication@unh.edu. 


\title{
A hard X-ray polarimeter for gamma-ray bursts and solar flares
}

\author{
Mark L. McConnell, James R. Ledoux, John R. Macri, and James M. Ryan \\ Space Science Center, University of New Hampshire, Durham, NH 03824
}

\begin{abstract}
We report on the development of a dedicated polarimeter design that is capable of studying the linear polarization of hard X-rays (50-300 keV) from $\gamma$-ray bursts and solar flares. This compact design, based on the use of a large area position-sensitive PMT (PSPMT), is referred to as GRAPE (Gamma-RAy Polarimeter Experiment). The PSPMT is used to determine the Compton interaction location within an array of small plastic scintillator elements. Some of the photons that scatter within the plastic scintillator array are subsequently absorbed by a small centrally-located array of $\mathrm{CsI}(\mathrm{Tl})$ crystals that is read out by an independent multi-anode PMT. One feature of GRAPE that is especially attractive for studies of $\gamma$-ray bursts is the significant off-axis response (at angles $>60^{\circ}$ ). The modular nature of this design lends itself toward its accomodation on a balloon or spacecraft platform. For an array of GRAPE modules, sensitivity levels below a few percent can be achieved for both $\gamma$-ray bursts and solar flares. Here we report on the latest results from the testing of a laboratory science model.
\end{abstract}

Keywords: X-ray, X-ray astronomy, gamma-ray, gamma-ray astronomy, polarimetry, polarization, gamma-ray burst, solar flare

\section{INTRODUCTION}

Although polarimetry has become a powerful tool for astronomers throughout much of the electromagnetic spectrum, progress in hard X-ray and $\gamma$-ray polarimetry has been slow. Many models for hard X-ray and $\gamma$-ray emission involve beams of highly energetic particles in extremely strong magnetic fields. A natural by-product of such an ordered emission geometry is linearly polarized photon emission. Linearly polarized emission is also expected in the case of scattered radiation, such as the Compton scattered emission seen in many X-ray sources. Many source models therefore predict significant polarization levels in the hard X-ray regime (defined here to be 50-300 keV). The level of polarization will depend on the precise emission geometry. In addition, the energy-dependence of the polarization can provide clues to the emission mechanisms that may be operating. Polarization measurements therefore have the potential to tell us something about both the mechanisms and source geometries responsible for the observed emissions. ${ }^{1}$ Despite this potential for useful scientific information, there has been limited progress in this field, largely due to the lack of sufficiently sensitive instrumentation. Here we describe the latest results from our efforts to develop a hard X-ray / soft $\gamma$-ray polarimeter that is particular well-suited to the study of $\gamma$-ray bursts and solar flares.

\subsection{Gamma-Ray Bursts}

In recent years, largely as a result of the observation of several X-ray, optical and radio afterglows, there has developed a growing consensus that classical $\gamma$-ray bursts (GRBs) are at cosmological distances. Such great distances imply that a typical GRB releases $10^{51}-10^{53}$ ergs or more within a time span of several seconds. The general picture that has emerged is one that describes the GRB phenomenom in terms of a relativistic fireball model. ${ }^{2}$ According to this scheme, the $\gamma$-rays are emitted when an ultra-relativistic energy flow is converted into radiation. The nature of the inner engine that releases this energy flow is still a subject of speculation. Once released, however, it is believed that the prompt gamma-ray emission is produced by internal shocks within the outgoing flow. Internal shocks are generated when one expanding shell overtakes another, slower-moving shell. An external shock arises when the outgoing flow runs into the local interstellar medium (ISM). The longer wavelength (soft X-ray, optical, radio) afterglow emission is believed to arise from the external shock.

Correspondence: E-mail: Mark.McConnell@unh.edu 
One common feature of many GRB models is that the energy release takes the form of jets that are directed along the rotation axis of the system. Several indirect arguments have been used to argue that such jets are required to explain the observations..$^{3-5}$ Since the energy budget of a given GRB depends heaviliy on assumptions about the extent to which the flow is jet-like, determining the reality and nature of jets in GRBs is becoming an important goal of future observations. The observation of optical polarization in GRB afterglows, such as that for GRB990510 ${ }^{6}$ has provided direct evidence for geometrical beaming of emission related to the external shocks. Although the measured level of optical polarization is small $(\sim 2 \%)$, several models predict levels of polarization as high as 10 or $20 \%$, depending on the angle between the observer and the jet axis. ${ }^{5,7-9}$ Optical polarization measurements, and the theoretical studies that have been motivated by such measurements, have provided a better insight into the nature of the GRB phenomena. The optical studies, however, probe only the external shock region. In the context of the canonical fireball model, measurements of the hard X-ray polarization during the prompt phase of the GRB promise to provide a similar probe of the internal shock region. Since the outgoing flow at the internal shock is expected to be more tightly collimated than the flow at the external shock (resulting from a continuous spreading of the jet as it progresses outward through the fireball), one can perhaps expect a somewhat higher level of hard X-ray polarization during the prompt phase of the GRB. Both inverse Compton and synchrotron emission have been proposed as viable emission mechanisms for GRBs. The spectral signatures of these two processes can be very similar, so it is very difficult to determine the responsible mechanism on the basis of spectral measurements alone. Energydependent polarization measurements, in principle, offer a possible solution. The degree of linear polarization of synchrotron emission, unlike inverse Compton emission, is independent of energy. This energy-dependence of the polarization (or lack thereof) may therefore be exploited as a means of identifying the emission mechanism responsible for the hard X-ray and $\gamma$-ray emission.

It is widely recognized that the soft $\gamma$-ray repeaters (SGR) represent a different class of phenomena than the classical GRBs. SGRs are short duration, soft-spectrum bursts with super-Eddington luminosities. The bulk of the emission is seen at energies below $100 \mathrm{keV}$. A total of four, perhaps five, such sources have now been identified. ${ }^{10}$ The prevailing view is that SGR outbursts involve emission from the vicinity of magnetars, neutron stars with magnetic fields in excess of $10^{14} \mathrm{G}$. Baring ${ }^{11}$ suggested that the softness of the events can be attributed to photon splitting in the extremely intense magnetic fields. One by-product of photon splitting is that the reprocessed photons would exhibit a polarization level of $\sim 25 \% .{ }^{11}$ Polarization measurements could therefore provide a test of the importance of photon splitting in SGRs.

\subsection{Solar Flares}

Polarization measures are also expected to be useful in determining the beaming (or directivity) of solar flare electrons, a quantity that may provide important clues about electron acceleration and transport. Although statistical studies have shed some light on the issue of electron beaming ${ }^{12-14}$ polarization studies are expected to provide detailed information on individual flares and perhaps even study time-dependent behavior within individual flares. Efforts to measure solar flare polarizations at energies below $30 \mathrm{keV}$ have so far met with limited success, in part due to contamination by thermal electron populations. ${ }^{15-18}$ Measurements at energies above $100 \mathrm{keV}$ are expected to be most useful, ${ }^{19}$ where we expect large linear polarizations of the electron bremstrahlung radiation due to the anisotropy of the electrons. ${ }^{20,21}$ The recently-launched RHESSI (Ramaty High Energy Solar Spectroscopic Imager) satellite ${ }^{22}$ will be able to measure polarization in the larger (X-class) solar flares. ${ }^{23}$ Unfortunately, it will be most sensitive at energies below $100 \mathrm{keV}$, where thermal emission may dominate. Measurements near $100 \mathrm{keV}$ and above may be required to provide an effective probe of the acceleration region in solar flares.

Many models of nonthermal (e.g., thick target) hard X-ray production predict a clear and significant polarization signal, with polarization levels $>10 \% \cdot{ }^{20,21,24-28}$ These predictions, while clearly testable, could be criticized on the grounds that the modeling assumptions they contain may be oversimplistic. For example, each model to date assumes a single, simple magnetic field structure about which the emitting electrons spiral. It could be argued that any real flare, particularly one sufficiently large to produce a signal of sufficient strength to enable a polarization measurement to be made, will in all probability contain a mix of structures that would average out any polarization signal present. However, observations from Yohkoh of large, simple magnetic 
structures even in large flares ${ }^{29}$ give support to the possibility that a statistically significant polarization signal could be produced in a large event.

\subsection{Experimental Status}

To date, the use of polarimetry in X-ray and $\gamma$-ray astronomy has been largely limited to energies below $\sim 30$ $\mathrm{keV} .{ }^{15,30-32}$ At higher energies, there are some instruments, such as COMPTEL ${ }^{33}$ and INTEGRAL, ${ }^{1}$ that have incorporated polarimetry into their design, but there have been only limited efforts to develop experiments whose primary function is to perform polarimetry. ${ }^{1,34-37}$ Results from COMPTEL have been inconclusive, due to systematic effects that are currently not understood. ${ }^{38}$ The IBIS experiment on INTEGRAL will have good polarization sensitivity, but the relatively small field-of-view ( $\sim 0.1$ steradian $)$ and limited telemetry may limit its usefulness in GRB studies. Our work concentrates on an optimized polarimeter design that, in part because of its large FoV, will be effective for both GRBs and solar flares. It will also be a viable technology for use in an imaging polarimeter configuration.

\section{COMPTON POLARIMETERS}

The basic physical process used to measure polarization in the $50-300 \mathrm{keV}$ energy range is Compton scattering, ${ }^{1}$ the cross-section for which is given by, ${ }^{39}$

$$
d \sigma=\frac{r_{0}^{2}}{2} d \Omega\left(\frac{\nu^{\prime}}{\nu_{o}}\right)^{2}\left(\frac{\nu_{o}}{\nu^{\prime}}+\frac{\nu^{\prime}}{\nu_{o}}-2 \sin ^{2} \theta \cos ^{2} \eta\right)
$$

where,

$$
\frac{\nu^{\prime}}{\nu_{o}}=\frac{1}{1+\left(\frac{h \nu_{o}}{m c^{2}}\right)(1-\cos \theta)}
$$

Here $\nu_{o}$ is the frequency of the incident photon, $\nu^{\prime}$ is the frequency of the scattered photon, $\theta$ is the scattering angle of the scattered photon measured from the direction of the incident photon, and $\eta$ is the azimuthal angle of the scattered photon measured from the plane containing the electric vector of the incident photon. For a given value of $\theta$, the scattering cross section for polarized radiation reaches a minimum at $\eta=0^{\circ}$ and a maximum at $\eta=90^{\circ}$. In other words, photons tend to be scattered at a right angle with respect to the incident electric field vector. In the case of an unpolarized beam of incident photons, there will be no net positive electric field vector and therefore no preferred azimuthal scattering angle $(\eta)$; the distribution of scattered photons with respect to $\eta$ will be uniform. However, in the polarized case, the incident photons will exhibit a net positive electric field vector and the distribution in $\eta$ will be asymmetric.

The ability to measure the polarization of the incident photon beam depends on the asymmetry ratio. ${ }^{40}$ This is defined to be the ratio between the maximum in the $\eta$ distribution to the minimum intensity in the $\eta$ distribution,

$$
R\left(\frac{\max }{\min }\right)=\frac{\left(\nu_{o} / \nu^{\prime}+\nu^{\prime} / \nu_{o}\right)}{\left(\nu_{o} / \nu^{\prime}+\nu^{\prime} / \nu_{o}-2 \sin ^{2} \theta\right)}
$$

This distribution is plotted as a function of scattering angle $(\theta)$ for various incident photon energies in Fig. 1. This plot shows that the asymmetry ratio is larger at lower energies and that events with scattering angles between $60^{\circ}$ and $120^{\circ}$ contain most of the polarization information. In general, a Compton scatter polarimeter consists of two detectors to determine the energies of both the scattered photon and the scattered electron. One detector, the scattering detector, provides the medium for the Compton interaction to take place. This detector must be designed to maximize the probability of there being a single Compton interaction with a subsequent escape of the scattered photon. This requires a low-Z material in order to minimize photoelectric interactions. The area of the scattering detector which is exposed to the photon beam is also an important 


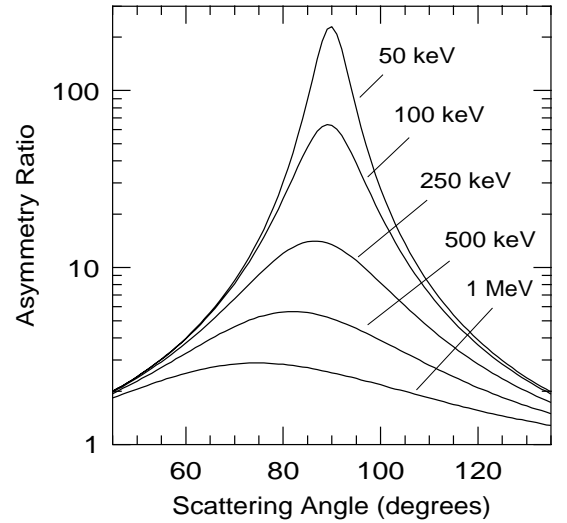

Figure 1: The asymmetry ratio (Eq. 3) for various incident photon energies.

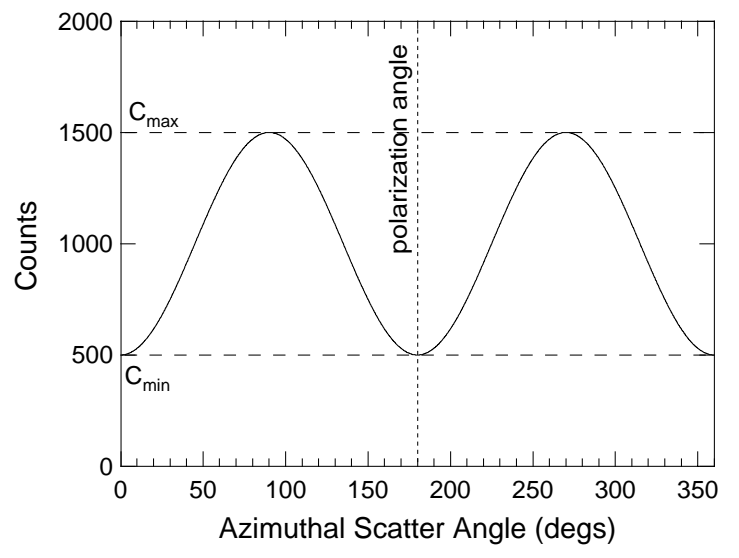

Figure 2: The modulation pattern produced by Compton scattering of polarized radiation. The minimum of the modulation pattern defines the plane of polarization of the incident flux. In this case, $\phi=180^{\circ}$.

factor in determining the effective area of the polarimeter. The primary purpose of the second detector, the calorimeter, is to aborb the full energy of the scattered photon.

The relative placement of the two detectors defines the scattering geometry. For incident photon energies below $100 \mathrm{keV}$, the azimuthal modulation of the scattered photons is maximized if the two detectors are placed at a right angle relative to the incident photon beam $\left(\theta=90^{\circ}\right.$; c.f., Fig. 1). The positioning of the two detectors must also be arranged relatively close to each other so that there is a finite solid angle for scattering to achieve the required detection efficiency. At the same time, a larger separation between the detectors provides more precise scattering geometry information. The accuracy with which the scattering geometry can be measured determines the ability to define the modulation pattern (Fig. 2) and therefore has a direct impact on the polarization sensitivity (see below). Here, one must compromise between total efficiency (small detector separation) and the ability to define the modulation pattern (large detector separation).

The ultimate goal of a Compton scatter polarimeter is to measure the azimuthal modulation pattern of the scattered photons. From Eq. 1, we see that the azimuthal modulation follows a $\cos 2 \eta$ distribution. More specifically, we can write the integrated azimuthal distribution of the scattered photons as,

$$
C(\eta)=A \cos \left(2\left(\eta-\phi+\frac{\pi}{2}\right)\right)+B
$$

where $\phi$ is the polarization angle of the incident photons. (In practice, a measured distribution must also be corrected for geometrical effects based on the corresponding distribution for an unpolarized beam. ${ }^{1}$ ) It is customary to define, as a figure-of-merit for the polarimeter, the polarization modulation factor ${ }^{1,30}$. For a given energy and incidence angle for an incoming photon beam, this can be expressed as,

$$
\mu_{p}=\frac{C_{p, \max }-C_{p, \min }}{C_{p, \max }+C_{p, \min }}=\frac{A_{p}}{B_{p}}
$$

where $C_{p, \max }$ and $C_{p, \min }$ refer to the maximum and minimum number of counts registered in the polarimeter, respectively, with respect to $\eta ; A_{p}$ and $B_{p}$ refer to the corresponding parameters in equation (4). In this case the $p$ subscript denotes that this refers to the measurement of a beam with unknown polarization. In order to determine the polarization of the measured beam, we need first to know how the polarimeter would respond to a similar beam, but with 100\% polarization. This can be done using Monte Carlo simulations. We then define a corresponding modulation factor for an incident beam that is $100 \%$ polarized, 


$$
\mu_{100}=\frac{C_{100, \text { max }}-C_{100, \text { min }}}{C_{100, \text { max }}+C_{100, \text { min }}}=\frac{A_{100}}{B_{100}}
$$

Then, following Novick, ${ }^{30}$ we can then use this result, in conjunction with the observed modulation factor $\left(\mu_{p}\right)$, to determine the level of polarization in a measured beam,

$$
P=\frac{\mu_{p}}{\mu_{100}}
$$

where $P$ is the measured polarization. The minimum detectable polarization (MDP) can be expressed as, ${ }^{30}$

$$
M D P(\%)=\frac{n_{\sigma}}{\mu_{100} S} \sqrt{\frac{2(S+B)}{T}}
$$

where $n_{\sigma}$ is the significance level (number of sigma), $S$ is the total source counting rate, $B$ is the total background counting rate and $T$ is the total observation time. Improved sensitivity to source polarization can be achieved either by increasing the modulation factor $\left(\mu_{100}\right)$ or by increasing the effective area of the polarimeter (thereby increasing the source counting rate, $S$ ).

\section{A MODULAR POLARIMETER DESIGN}

We have developed a modular polarimeter design (GRAPE Gamma RAy Polarimeter Experiment) that places an entire device on the front end of a single 5-inch diameter position-sensitive PMT (PSPMT). ${ }^{41-45}$ The design (Fig. 3) incorporates an array of plastic scintillator elements to provide the improved spatial resolution in the scattering medium and to improve the rejection of multiple scatter events. Each plastic scintillator element is optically-isolated with a cross sectional area of $5 \times 5 \mathrm{~mm}^{2}$. The plastic elements are arranged in the form of an annulus having an outside diameter of $10 \mathrm{~cm}$ (corresponding to the sensitive area of the Hammatsu R3292 5 -inch PSPMT). The central portion of the annulus is large enough to insert a small $2 \times 2$ array of $1 \mathrm{~cm} \mathrm{CsI(Tl)}$ scintillators. The CsI(Tl) scintillators are coupled to their own independent multi-anode PMT (MAPMT) for the energy measurement and signal timing. In the design depicted in Fig. 3, both the plastic and CsI(Tl) elements have a depth of $5 \mathrm{~cm}$.

To be recorded as a polarimeter event, an incident photon Compton scatters from one (and only one) of the plastic array elements into the central calorimeter. The incident photon energy can be determined from the sum of the energy losses in both detectors. The azimuthal scattering angle $(\eta)$ can be determined by the azimuthal angle of the associated plastic element. When the polarimeter is arranged so that the incident flux is parallel to the symmetry axis, unpolarized radiation will produce an axially symmetric coincidence rate. If, on the other hand, the incident radiation is linearly polarized, then the coincidence rate will show an azimuthal asymmetry whose phase depends on the position angle of the incident radiation's electric field vector and whose magnitude depends on the degree of polarization. The polarization signature therefore comes from the azimuthal scatter angle distribution.

A series of Monte Carlo simulations have been used to determine the charcteristics of this design. These simulations assume that we are able to uniquely identify which plastic scintillator element is involved in the event. The small cross-sectional area of each scintillator element ensures that practically all multiple scatter events are rejected. The energy threshold levels, particularly in the scattering elements, have a significant influence on the performance of the polarimeter at low energies. For the simulations, we have assumed a threshold energy of $15 \mathrm{keV}$ in both the plastic and CsI scintillators.

Fig. 4 illustrates the nature of the polarimeter data. In this case, the data are from Monte Carlo simulations. The first panel shows the polarization response to a fully polarized monoenergetic beam of $150 \mathrm{keV}$ photons vertically incident on the front surface of the polarimeter. This distribution includes not only the intrinsic modulation pattern due to the Compton scattering process, but it also includes geometric effects related to the specific layout of the detector elements within the polarimeter and the associated quantization of possible 

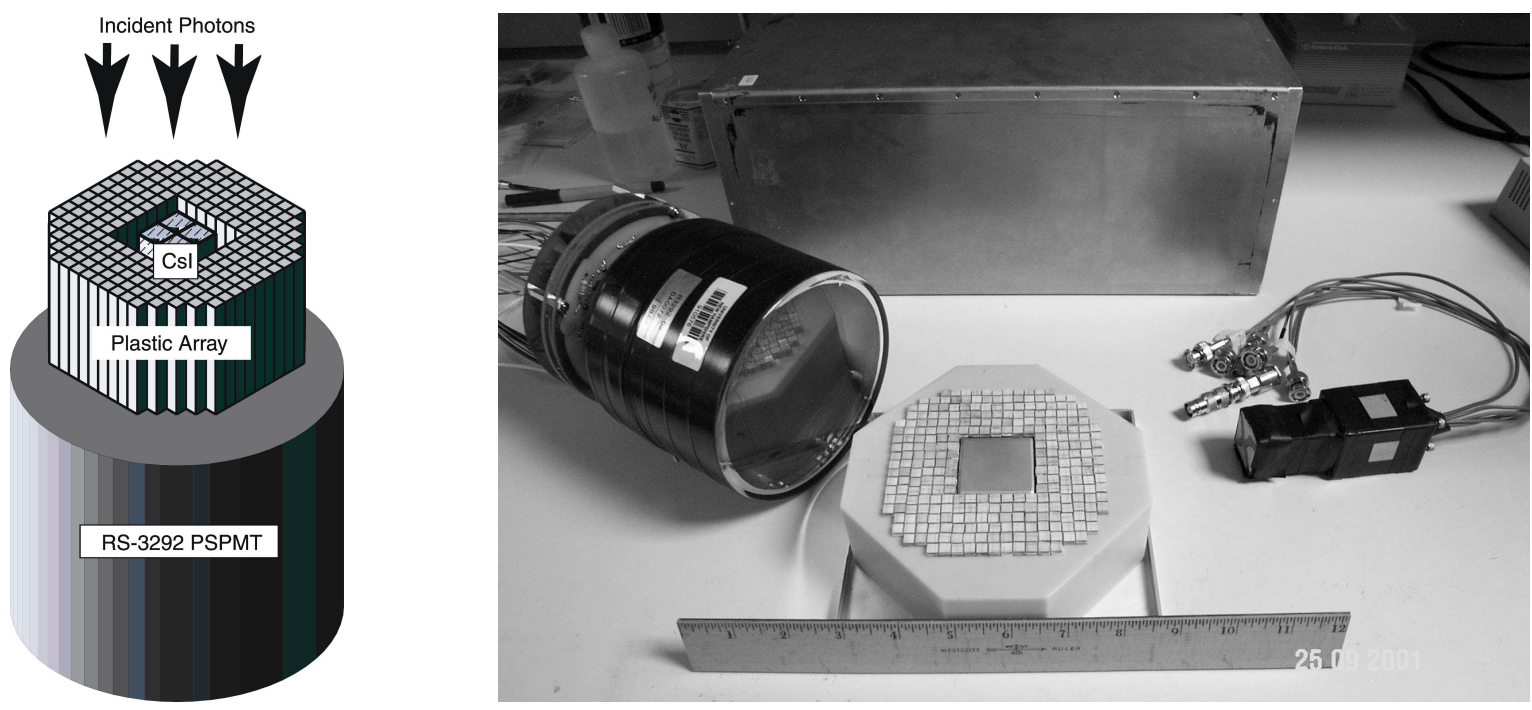

Figure 3: Left: The GRAPE polarimeter design showing the layout of the plastic scintillator elements and $\mathrm{CsI}(\mathrm{Tl})$ elements on the front surface of a PSPMT. Not shown here is the 4-element multianode PMT used for readout of the $\mathrm{CsI}(\mathrm{Tl})$ array. Right: The components of the laboratory science model. The aluminum box is the housing for the entire assembly.
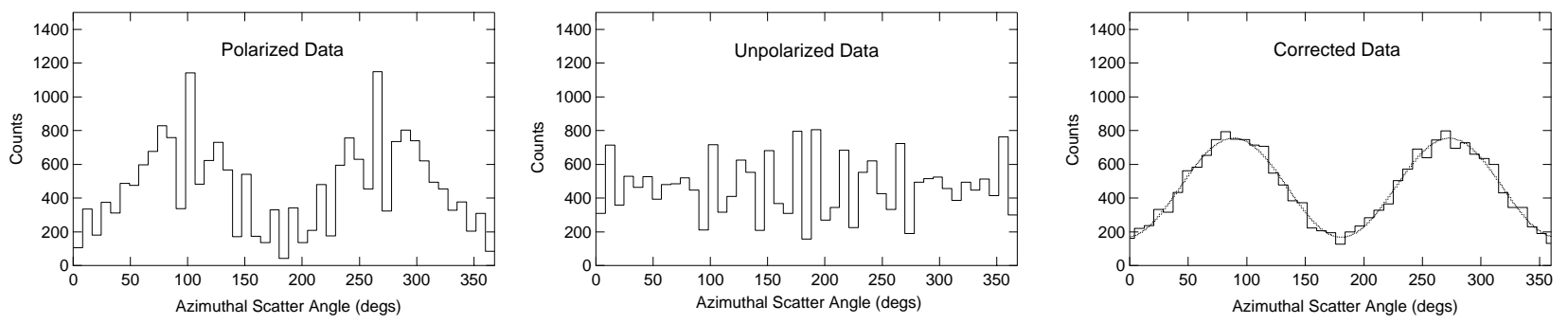

Figure 4: Simulated polarimeter data showing how the measured data is corrected for intrinsic geometric effects to extract the true modulation pattern (see text). These data correspond to the response of the GRAPE design to a monoenergetic beam of $150 \mathrm{keV}$ photons incident at $0^{\circ}$ (vertical incidence).

scatter angles. The geometric effects can be more clearly seen in the case of an incident beam that is completely unpolarized, as shown in the second panel of Fig. 4. (In practice, for analyzing real data, this unpolarized distribution would be determined by simulations rather than by direct measurements.) To extract the true distribution of polarized events, we divide the polarized distribution by the unpolarized distribution and normalize by the average of the unpolarized distribution. Only when we correct the raw data in this fashion do we clearly see the $\cos 2 \eta$ modulation pattern that is expected (the third panel of Fig. 4).

Simulated data have also been used to generate the data shown in Figs. 5 and 6, which show the effective area and modulation factor, respectively, as a function of incident photon energy. In both cases, are shown the results for two different detector depths $-5.08 \mathrm{~cm}$ and $7.62 \mathrm{~cm}$. Although the deeper detector clearly presents an advantage in terms of effective area, the varying detector depth appears to have little influence on the modulation factor. In practice, the advantage of increased effective area for a deeper detector must be offset by the increase in background as well as the decrease in light collection efficiency (with its consequent effects on the detector threshold).

One feature of the GRAPE design that is especially attractive for studies of GRBs is the significant off-axis polarization response. This can be seen in Fig. 7, which is based on simulations with a detector depth of 5.08 $\mathrm{cm}$. The exposed geometric area (and, hence, the effective area) of the detector remains relatively constant at 


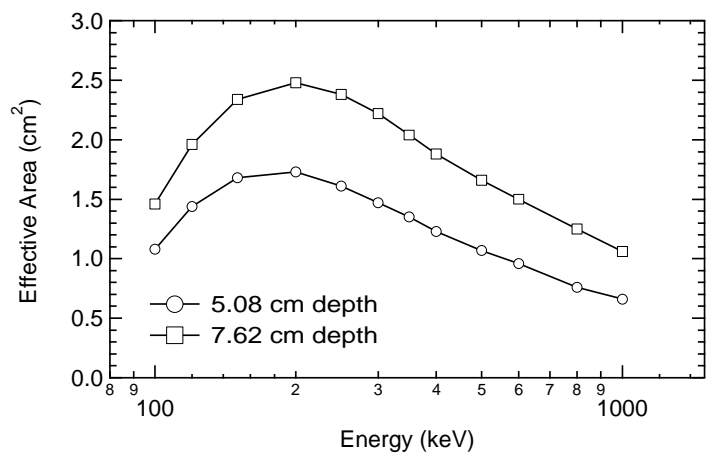

Figure 5: The effective area as a function of energy for a single GRAPE module with detector depths of $5.08 \mathrm{~cm}$ and $7.62 \mathrm{~cm}$.

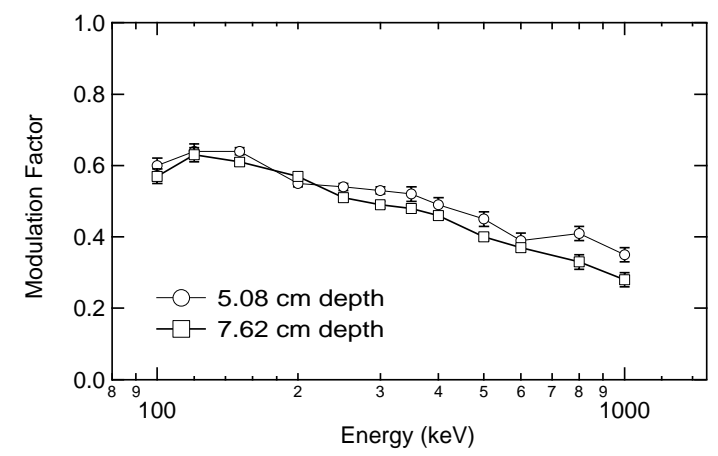

Figure 6: The modulation factor as a function of energy for a single GRAPE module with detector depths of $5.08 \mathrm{~cm}$ and $7.62 \mathrm{~cm}$.

large angles. Although there is a significant decrease in the modulation factor at large angles, there remains significant polarization response even at incidence angles as large as $60^{\circ}$.

\subsection{Polarization Sensitivity Estimates}

In practice, the GRAPE design would be used in the context of an array of polarimeter modules. Such an array could be made an integral part of either a long-duration balloon payload or an Earthorbiting spacecraft payload. To assess the capabilities of the GRAPE design for doing useful GRB studies, we have estimated the polarization sensitivity of a balloon payload, using Eq. 8 to calculate the $3 \sigma$ minimum detectable polarization (MDP). The double-scatter nature of this experiment implies a relatively low background. The dominant source of background will be the true coincidence rate (i.e., those photon events which actually scatter from the plastic scattering elements into the calorimeter). Our sensitivity calculations assume that the entire experiment is surrounded by an anticoincidence shield that effectively eliminates the

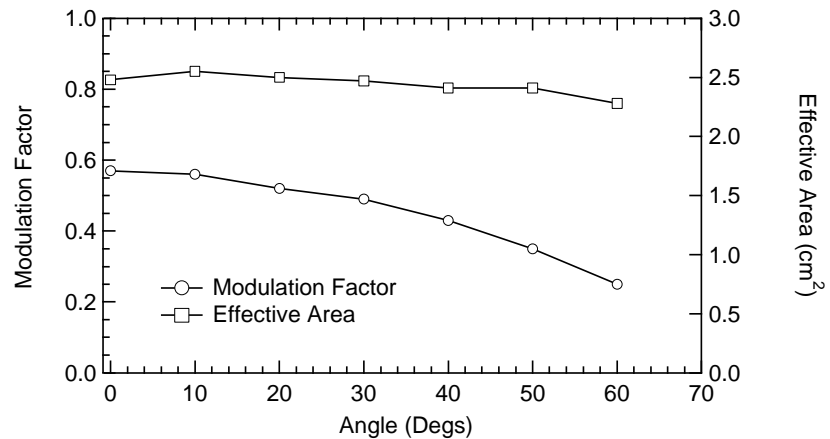

Figure 7: The modulation factor and effective area of a single GRAPE module at $200 \mathrm{keV}$ for various incidence angles. The polarimeter maintains good response out to $60^{\circ}$ incidence angles. background except for that which enters the open aperture. We assume an open aperture of $\pi$ sr and have used data from Gehrels ${ }^{46}$ to estimate the magnitude of the atmospheric background flux.

For the estimation of GRB sensitivity, we assume an array of 36 modules (which could easily fit within an area of $1 \mathrm{~m}^{2}$ ), each with a detector thickness of $12.7 \mathrm{~cm}$. The incident GRB spectrum is asumed to be represented by an $E^{-1.5}$ power law spectrum in the $50-300 \mathrm{keV}$ energy band. The fluence level is defined as that integrated over the 50-300 keV energy range. We have calculated the MDP for bursts of varying fluence and duration. For a given fluence level, there is greater polarization sensitivity for the shorter events, since they involve a lower level of background. The results of these calculations for the integrated 50-300 keV energy band are shown in Table 1. The observed rate of events is based on the fraction of events at a given fluence level in the BATSE 4B catalog ${ }^{47}$ and the fact that BATSE observed about one event per day with its $\sim 2 \pi$ sr effective FoV. These data indicate that GRAPE could do useful science with 50-300 keV GRB fluences as low as $3 \times 10^{-6} \mathrm{ergs} \mathrm{cm}^{-2}$. During the course of a 100-day balloon flight we could expect about ten events which provide a polarization sensitivity of better than $10 \%$ in the integrated $50-300 \mathrm{keV}$ energy band and about 25 
events which provide a polarization sensitivity of better than $\sim 30 \%$. For the stronger events, we could study the energy-dependence of the polarization.

Table 1: Minimum Detectable GRB Polarization (MDP), 50-300 keV

\begin{tabular}{|c|c|c|c|}
\hline \multirow{2}{*}{$\begin{array}{c}50-300 \mathrm{keV}^{\text {Fluence }} \\
\mathrm{ergs} \mathrm{cm}^{-2}\end{array}$} & \multicolumn{2}{|c|}{$M D P, 3 \sigma$} & Observed Rate \\
\hline & $\Delta \mathrm{T}=10 \mathrm{sec}$ & $\Delta \mathrm{T}=100 \mathrm{sec}$ & $N>$ Fluence \\
\hline $1 \times 10^{-4}$ & $2.8 \%$ & $2.9 \%$ & 1 every 320 days \\
\hline $5 \times 10^{-5}$ & $3.9 \%$ & $4.4 \%$ & 1 every 80 days \\
\hline $1 \times 10^{-5}$ & $9.3 \%$ & $13.7 \%$ & 1 every 10 days \\
\hline $5 \times 10^{-6}$ & $14.0 \%$ & $24.4 \%$ & 1 every 6 days \\
\hline $3 \times 10^{-6}$ & $19.4 \%$ & $38.6 \%$ & 1 every 4 days \\
\hline $1 \times 10^{-6}$ & $43.2 \%$ & - & 1 every 2 days \\
\hline
\end{tabular}

We have made similar sensitivity estimates for solar flares, in this case a 16 element array of GRAPE modules. ${ }^{45}$ It is difficult to make specific predictions about the sensitivity of such an array to any one flare due to the unique nature of each individual flare event. We have, however, estimated the polarization sensitivity based on the SMM-measured spectrum for the flare of 7 June 1980. The resulting sensitivity estimates indicate that an array of 16 modules would provide a minimum detectable polarization (MDP) of less than $1 \%$ in the integrated 50-300 keV energy range for some M-class flares and all X-class flares. Alternatively an array of four modules would provide a MDP of $1 \%$ or better in the integrated 50-300 keV energy range for all X-class flares. MDP levels of a few percent should be sufficient to test various models for hard X-ray polarization in solar flares.

\section{LABORATORY SCIENCE MODEL}

We have fabricated of a laboratory science model based on the modular GRAPE design (the components of which are shown in Fig. 3). The plastic scintillator array is composed of individual pieces of Bicron BC-404 scintillator. Each $5 \mathrm{~mm} \times 5 \mathrm{~mm} \times 50 \mathrm{~mm}$ scintillator element is individually wrapped in Tyvek to maximize light collection efficiency and to provide optical isolation. A thin layer of Kapton tape was then used to hold the wrapping in place. A thin aluminum housing encloses both the PSPMT and the plastic scintillator array. The calorimeter detector assembly is a $2 \times 2$ array of $1 \mathrm{~cm} \mathrm{CsI(Tl)} \mathrm{elements} \mathrm{coupled} \mathrm{to} \mathrm{a} \mathrm{MAPMT} \mathrm{(Hamamatsu}$ R5900 with a $2 \times 2$ anode array) and enclosed within its own, separate light-tight housing. During operations, the calorimeter detector assembly is inserted into a central well in the PSPMT / plastic scintillator housing. Data processing and acquisition is achieved using a combination of NIM and CAMAC modules, with the final data recorded via a SCSI interface on a Macintosh computer.

Our initial laboratory testing makes use of a charge division network for the PSPMT (Hammamatsu R3292) that provides a weighted average of the spatial distribution of the measured light output using only four signals (two signals in $x$ and 2 signals in $y$ ). In principle, more precise information regarding the distribution of energy deposits within the plastic arrays can be derive from using all 56 (28- $x$ plus 28-y) anode signals from the PSPMT. Our current plan is to implement an intermediate approach using only fourteen (7-x plus 7-y) anode wire sections. Such an approach has succeeded in resolving individual $3 \mathrm{~mm}$ YAP crystal elements using a center-of-gravity calculation for determining the interaction location. ${ }^{48}$ The utility of this readout scheme for rejecting multiple scatter events will be investigated. Given the mean free path of photons in plastic $(6 \mathrm{~cm}$ at $100 \mathrm{keV}$ ), we expect that a high level of multiple scatter event rejection can be achieved with the fourteen channel readout scheme, thus minimizing the required number of electrical channels.

We have carried out an LED mapping of the PSPMT spatial response using the four channel readout. The LED was stepped through a sequence of spatial locations with a fixed grid spacing of $2.5 \mathrm{~mm}$. The array of 


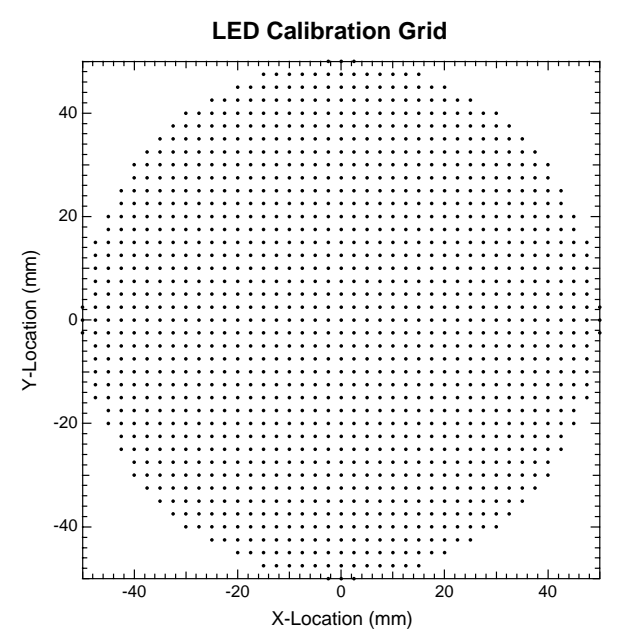

Figure 8: The array of locations used for the LED mapping of the PSPMT. The grid spacing is 2.5 $\mathrm{mm}$, covering the entire area of the $100 \mathrm{~mm}$ diameter sensitive area of the PSPMT cathode.

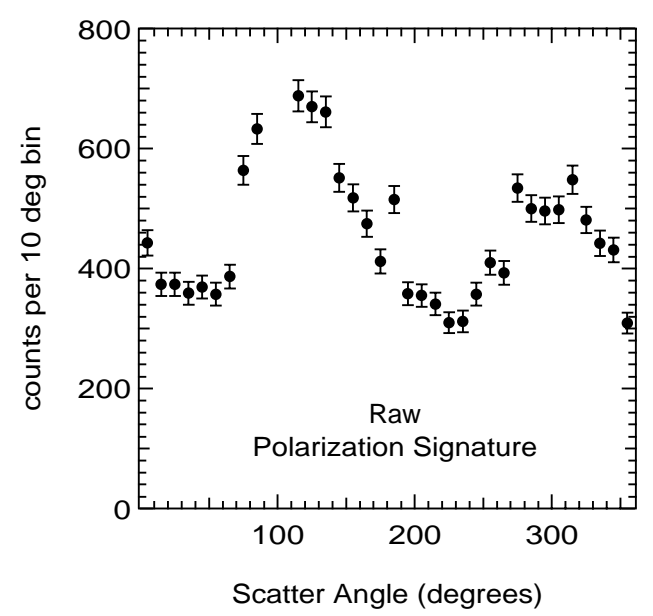

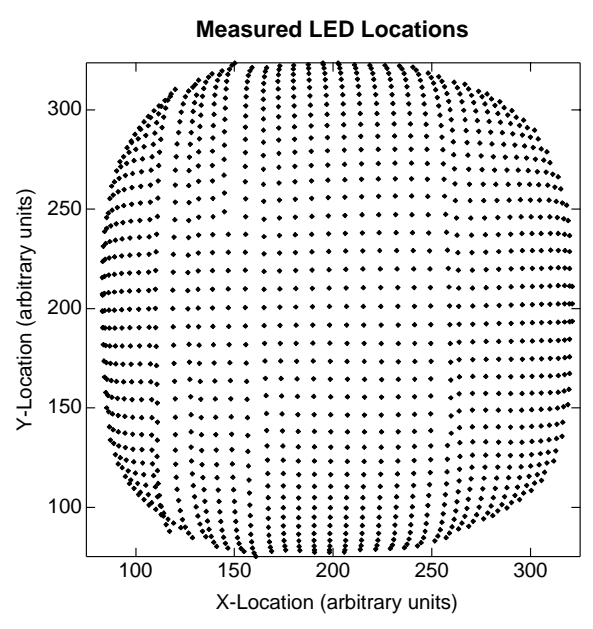

Figure 9: The array of measured locations (relative coordinates) in the LED mapping of the PSPMT. Note the significant distortions near the edge of the PSPMT.

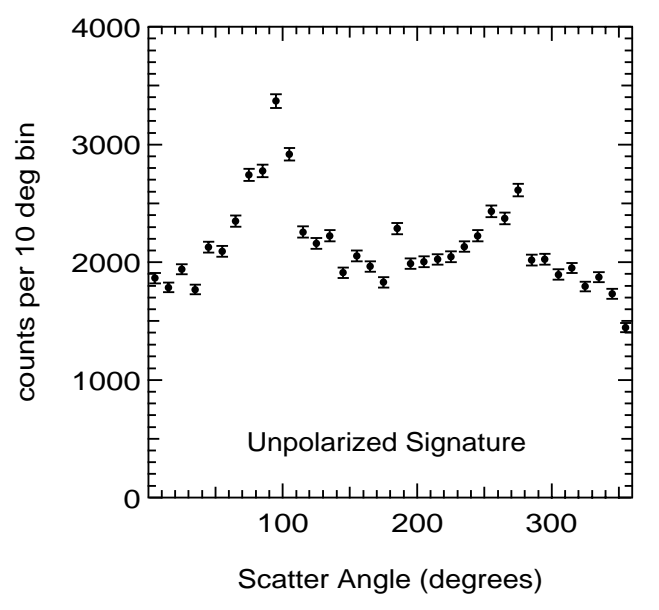

Figure 10: Calibration data from the GRAPE science model. The figure on the left shows the raw polarization signature derived from the polarized beam (with an incident energy of $288 \mathrm{keV}$ ). The figure shows the corresponding signature for a beam that is unpolarized (incident energy $356 \mathrm{keV}$ ).

measured grid locations is shown in Fig. 8. The distribution of measured locations, as mapped onto a relative coordinate grid, are shown in Fig. 9. Note the distortions of the grid pattern that are evident near the edge of the PSPMT. The results of the LED calibration are now routinely incorporated into the processing of data that are collected from the science model. For each event, the measured location in the plastic array is converted into a true location using the LED calibration data. This process removes the distortions that are intrinsic to the PSPMT and its readout. This results in more accurate event locations within the plastic array and a corresponding improvement in the polarization signal.

In order to perform laboratory calibrations of the assembled polarimeter, we generated partially polarized photons by scattering photons from a radioactive calibration source. In particular, we used photons from radioactive ${ }^{137} \mathrm{Cs}(662 \mathrm{keV})$ Compton scattered off a block of plastic scintillator at a nominal angle of $\sim 90^{\circ}$. This resulted in a beam of photons that is linearly polarized at a level between 50 and $60 \% .{ }^{42}$ The use of plastic 

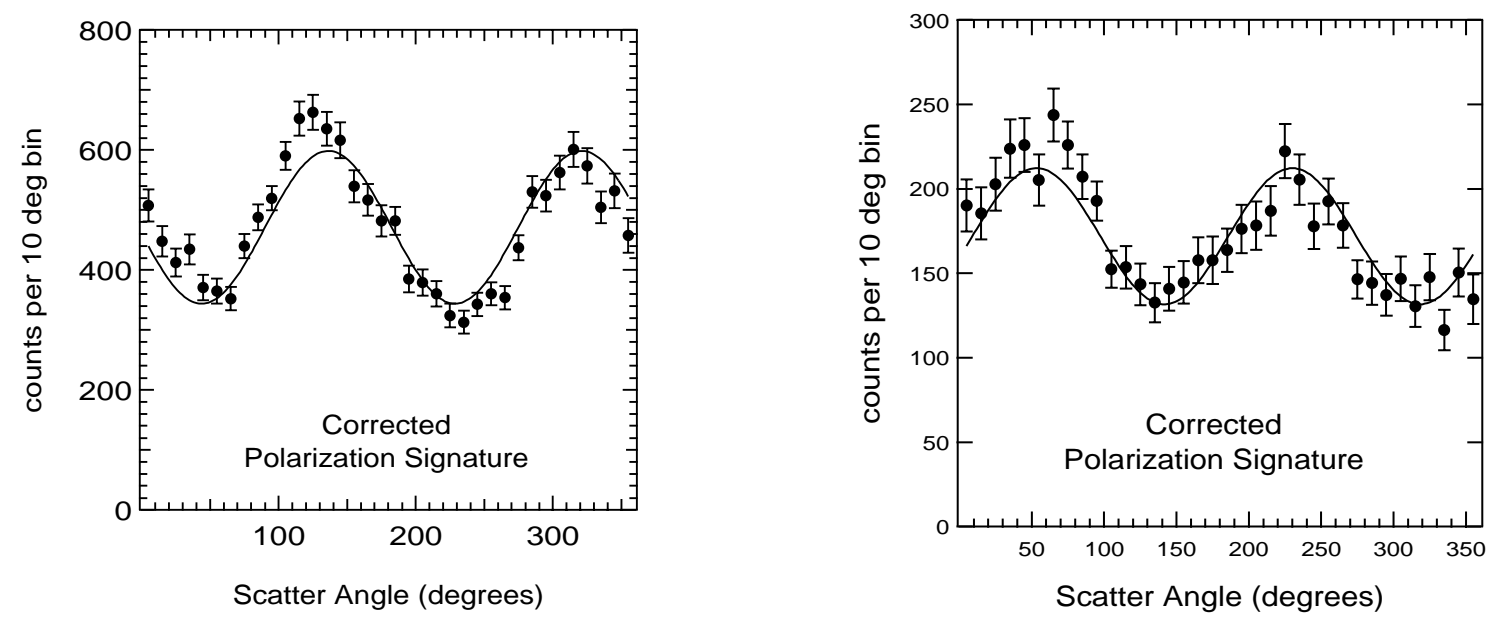

Figure 11: The final polarization signals derived from calibration runs at two different polarization angles (differing by $\left.\sim 90^{\circ}\right)$. The data have been fit to a function of the form $\cos 2 \eta$. The figure on the left corresponds to the data of Fig. 10.

scintillator has the added advantage of providing electronic tagging of the scattered photons. The requirement of a three-way coincidence between the scattering block, the PSPMT and the MAPMT ensures an extremely low (negligible) background. The use of a ${ }^{137} \mathrm{Cs}$ source means that the photons that are incident on the GRAPE science model have an energy of $\sim 288 \mathrm{keV}$. To obtain the needed comparison measurements with an unpolarized beam, we use $356 \mathrm{keV}$ photons directly from a ${ }^{133} \mathrm{Ba}$ source (i.e, with no initial scattering).

The data from a set of polarized/unpolarized runs is shown in Fig. 10. Fig. 11 shows the final polarization signature for two different polarization angles. The figure on the left in Fig. 11 corresponds to the data of Fig. 10. The right hand figure of Fig. 11 shows the same result derived from an incident beam whose polarization angle has been shifted by $\sim 90^{\circ}$. The data have been fit using a function of the form $\cos 2 \eta$. The fit parameters, along with simulated modulation factors for a fully polarized beam, can be used to derive both the level of linear polarization and the polarization angle. The polarization levels measured from these data are $P=51( \pm 3) \%$ and $P=44( \pm 4) \%$, respectively. These results are consistent with the expected polarization level of $P=50-60 \%$. The difference in polarization angle (corresponding shift in phase between the two polarization signals in Fig. 11) was determined to be $\phi=101( \pm 5)^{\circ}$, a result that is also consistent with the accuracy of the experimental setup.

\section{SUMMARY}

We are continuing our development of the GRAPE science model. We are currently completing a series of tests using the charge division readout of the PSPMT and are making preparations to develop an improved readout scheme that makes more complete use of the available anode signals. In addition to its potential for studying transient sources, the GRAPE design might also be useful in the context of an imaging polarimeter. For example, a GRAPE element or array of elements could be used with a rotation modulation collimator to achieve arc-second angular resolution. Such an approach is not unlike that employed for hard X-ray imaging (without polarization capability) by the RHESSI mission.

\section{ACKNOWLEDGMENTS}

This work is currently supported by NASA grant NAG5-5324.

\section{REFERENCES}

1. F. Lei, A.J. Dean and G.L. Hills, "Compton scatter polarimetry in gamma-ray astronomy," Space Sci. Rev. 82, pp. 309-388, 1997. 
2. T. Piran, "Gamma-ray bursts and the fireball model," Phys. Rept. 314, pp. 575-667, 1999.

3. J. E. Rhoads, "The dynamics and light curves of beamed gamma-ray burst afterglows," Ap. J. 525, pp. 737-749, 1999.

4. T. Nakamura, "Multiple sub-jet model of gamma ray bursts and possible origin of X-ray precursors and post-cursors," Ap. J. Letters 534, pp. L159-L162, 2000.

5. R. Sari, "Linear Polarization and Proper Motion in the Afterglow of Beamed Gamma-Ray Bursts," Ap. J. 524, pp. L43-L46, 1999.

6. S. Covino, et al., "GRB 990510: linearly polarized radiation from a fireball," Astron. Astrophys. 348, pp. L1-L4, 1999.

7. A. Gruzinov, "Strongly polarized optical afterglows of gamma-ray bursts," Ap. J. 525, pp. L29-L31, 1999.

8. A. Gruzinov, and E. Waxman, "Gamma-ray burst afterglow: polarization and analytic light curves," $A p$. J. 511, pp. 852-861, 1999.

9. G. Ghisellini and D. Lazzati, "Polarization lightcurves and position angle variation of beamed gamma-ray bursts," MNRAS 309, pp. L7-L11, 1999.

10. K. Hurley, "The $4.5 \pm 0.5$ soft gamma repeaters in review," in AIP Conf. Proc. 510, The Fifth Compton Symposium, M.L. McConnell and J.M. Ryan, ed., pp. 515-522, AIP, New York, 2000.

11. M. Baring, "Photon-splitting limits to the hardness of emission in strongly magnetized soft gamma repeaters," Ap. J. 440, pp. L69-L72, 1995.

12. S. V. Bogovlov, et al., "Directionality of solar flare hard X-rays: Venera 13 observations," Sov. Astron. Lett. 11, pp. 322-324, 1985.

13. W. T. Vestrand, D. J. Forrest, E. L. Chupp, E. Rieger, and G. Share, "The directivity of high-energy emission from solar flares - Solar Maximum Mission observations," Ap. J. 322, pp. 1010-1022, 1987.

14. S. R. Kane, et al., "Directivity of $100 \mathrm{keV}-1 \mathrm{MeV}$ photon sources in solar flares," Ap. J. 326, pp. 1017-1031, 1988.

15. I. P. Tindo, V. D. Ivanov, S. L. Mandel'stam, and A. I. Shuryghin, "New measurements of the polarization of X-ray solar flares," Solar Phys. 24, pp. 429-433, 1972.

16. M. P. Nakada, W. M. Neupert, and R. J. Thomas, "Polarization results of solar X-rays from OSO-7," Solar Phys. 37, pp. 429-435, 1974.

17. I. P. Tindo, A. I. Shuryghin, and W. Steffen, "The polarization of X-ray emission of some solar flares in July 1974," Solar Phys. 46, pp. 219-227, 1976.

18. L. J. Tramiel, G. A. Channan, R. and Novick, "Polarization evidence for the isotropy of electrons responsible for the production of 5-20 keV X-rays in solar flares," Ap. J. 280, pp. 440-447, 1984.

19. G. Chanan, A.G. Emslie, and R. Novick, "Prospects for solar flare X-ray polarimetry," Solar Phys. 118, pp. 309-319, 1988.

20. T. Bai and R. Ramaty, "Backscatter, anisotropy, and polarization of solar hard X-rays," Ap. J. 219, pp. 705-726, 1978.

21. J. Leach and V. Petrosian, "The impulsive phase of solar flares. II - Characteristics of the hard X-rays," Ap. J. 269, pp. 715-727, 1983.

22. R. P. Lin et al., "The High Energy Solar Spectroscopic Imager (HESSI) Mission," in ASP Conf. Ser. 206, High Energy Solar Physics - Anticipating HESSI, R. Ramaty and N. Mandzhavidze, ed., pp. 1-12, ASP, San Francisco, 2000.

23. M. L. McConnell, et al., "Hard X-ray polarimetry with the Ramaty High Energy Solar Spectroscopic Imager," to be published in SPIE Proc 4843, 2002.

24. J. C. Brown, "The directivity and polarisation of thick target X-ray bremsstrahlung from solar flares," Solar Phys. 26, pp. 441-459, 1972.

25. Ju. E. Charikov, A. B. Guzman, and I. V. Kudryavtsev, "Hard X-ray emission of solar flares and nonstationary kinetics of electron beams," Astron. Astrophys. 308, pp. 924-928, 1996.

26. A. G. Emslie and L. Vlahos, "Radiation signatures from a locally energized flaring loop," Ap. J. 242, pp. 359-373, 1980. 
27. S. H. Langer and V. Petrosian, "Impulsive solar X-ray bursts. III - Polarization, directivity, and spectrum of the reflected and total bremsstrahlung radiation from a beam of electrons directed toward the photosphere," Ap. J. 215, pp. 666-676, 1977.

28. V. V. Zharkova, J. C. Brown, and D. V. Syniavskii, "Electron beam dynamics and hard X-ray bremsstrahlung polarization in a flaring loop with return current and converging magnetic field," Astron. Astrophys. 304, pp. 284-295, 1995.

29. S. Masuda, T. Kosugi, H. Hara, T. Sakao, K. Shibata, and S. Tsuneta, "Hard X-ray sources and the primary energy-release site in solar flares," Publ. Astron. Soc. Japan 47, pp. 677-689, 1995.

30. R. Novick, "Stellar and solar X-ray polarimetry," Space Sci. Rev. 18, pp. 389-408, 1975.

31. J. R. P. Angel, R. Novick, P. Vanden Bout, and R. Wolf, "Search for X-ray polarization in Sco X-1," Phys. Rev. Letters 22, pp. 861-865, 1969.

32. M. C. Weisskopff, R. F. Elsner, R. Novick, P. Kaaret, and E. Silver, "Predicted performance of the lithium scattering and graphite crystal polarimeter for the Spectrum-X-Gamma mission," Proc. SPIE 1343, pp. 457-468, 1990.

33. V. Schönfelder, et al., "Instrument description and performance of the Imaging Gamma-Ray Telescope COMPTEL aboard the Compton Gamma-Ray Observatory," Ap. J. Supp. 86, pp. 657-692, 1993.

34. H. Sakurai, M. Noma, and H. Niizeki, "A hard X-ray polarimeter utilizing Compton scattering," Proc. SPIE 1343, pp. 512-518, 1990.

35. E. Costa, M. N. Cinti, M. Feroci, G. Matt, and M. Rapisarda, "Scattering polarimetry for x-ray astronomy by means of scintillating fibers," Proc. SPIE 2010, pp. 45-56, 1993.

36. T.L. Cline, et al., A gamma-ray burst polarimeter study, in Proceedings of the 25th Internat. Cosmic Ray Conf., 5, pp. 25-28, 1997.

37. E. Caroli, et al., "Hard X-ray polarimetry with a thick CdTe position sensitive spectrometer," Proc. SPIE 4140, pp. 573-583, 2000.

38. F. Lei, G. L. Hills, A. J. Dean, and B. M. Swinyard, "Characteristics of COMPTEL as a polarimeter and its data analysis.," Astron. Astrophys. Supp. C120, pp. 695-698, 1996.

39. R. D. Evans, The Atomic Nucleus, McGraw-Hill, New York, 1958.

40. E. L. Chupp, Gamma-Ray Astronomy, D. Reidel Publishing Co., Boston, 1976.

41. M.L. McConnell, D.J. Forrest, J. Macri, J.M. Ryan, and W.T. Vestrand, "Development of a hard X-ray polarimeter for gamma-ray bursts," in AIP Conf. Proc. 428, Gamma-Ray Bursts, C.A. Meegan and P. Cushman, ed., pp. 889-893, AIP, New York, 1998.

42. M.L. McConnell, D.J. Forrest, J. Macri, M. McClish, M. Osgood, J.M. Ryan, W.T. Vestrand and C. Zanes "Development of a hard X-ray polarimeter for solar flares and gamma-ray bursts," IEEE Trans. Nucl. Sci. 45, pp. 910-914, 1998.

43. M.L. McConnell, J.R. Macri, M. McClish, J. Ryan, D.J. Forrest and W.T. Vestrand, "Development of a hard X-ray polarimeter for astrophysics," IEEE Trans. Nucl. Sci 46, pp. 890, 1999.

44. M.L. McConnell, J.R. Macri, M. McClish, and J. Ryan, "Recent laboratory tests of a hard X-ray solar flare polarimeter," SPIE Proc. 3764, pp. 70-78, 1999.

45. M. L. McConnell, J. R. Macri, and J. M. Ryan, "A modular hard X-ray polarimeter for solar flares," in ASP Conf. Ser. 206, High Energy Solar Physics - Anticipating HESSI, R. Ramaty and N. Mandzhavidze, ed., pp. 280-283, ASP, San Francisco, 2000.

46. N. Gehrels, "Instrumental background in balloon-borne gamma-ray spectrometers and techniques for its reduction," Nucl. Instr. Meth. A239, pp. 324-349, 1985.

47. W. S. Paciesas, et al., "The Fourth BATSE Gamma-Ray Burst Catalog (Revised)," Ap. J. Supp. 122, pp. 465-495, 1999.

48. R. Wojcik, S. Majewski, B. Kross, D. Steinbach, and A.G., "High spatial resolution gamma imaging detector based on a 5" diameter R3292 Hamamatsu PSPMT," IEEE Trans. Nucl. Sci. 45, pp. 487-491, 1998. 